\title{
Per una Teologia in dialogo: il sapere di Dio e i saperi dell'vomo
}

Piero Coda

\section{Resumo}

Il tema dell'articolo è l'apporto che la teologia può ed è chiamata a offrire oggi al dialogo tra i saperi. Questo tema è svolto in due momenti: nel primo, si richiama qualche dato sullo statuto epistemico della teologia, sulla sua singolarità e sull'ambito del conoscere che le è proprio; nel secondo, si affronta la questione del rapporto tra la teologia e gli altri saperi, disegnando alcune coordinate di una "mappa dei saperi" che sia coerente con l'esercizio dell'intelligenza in Cristo. Si tratta, nel rispetto dell'identità e del metodo di ogni sapere, di muovere dal condiviso presupposto secondo cui lo statuto epistemico di ogni scienza non riguarda soltanto le condizioni interne per un suo corretto attuarsi, ma anche quelle relazionali relative al rapporto di essa con le altre forme del sapere, che esprimono ciascuna - al proprio specifico livello - qualcosa della persona e attingono ciascuna, per la sua parte, qualcosa di vero circa la realtà.

Parole-chiavi: Dialogo, Epistemologia, Metodo, Sapere, Scienza, Teologia. 


\section{Resumo}

O tema do presente artigo diz respeito à aproximação que a teologia pode e é chamada a oferecer, hoje, ao diálogo entre os saberes. Este tema desenvolve-se em dois momentos: no primeiro, evocam-se alguns pontos sobre o estatuto epistêmico da teologia, sobre sua singularidade e sobre o âmbito do conhecer que lhe é próprio; no segundo, enfrenta-se a questão da relação entre a teologia e os outros saberes, traçando algumas coordenadas de um "mapa do saber" que seja coerente com o exercício da inteligência em Cristo. Trata-se, no respeito da identidade e do método de cada saber, de mover da aceitação do pressuposto segundo o qual o estatuto epistêmico de cada ciência não diz respeito somente às condições internas para seu correto atuar, mas também aquelas relacionais relativas à relação desta com as outras formas de saber, que exprimem, cada uma - no seu próprio nível - algo da pessoa e obtém cada uma, por sua vez, alguma coisa de verdadeiro sobre a realidade.

Palavras-chave: Ciência, Diálogo, Epistemologia, Método, Saber, Teologia.

L'obiettivo che mi propongo è di offrire qualche spunto di riflessione intorno all'apporto che la teologia può ed è chiamata a offrire al dialogo tra $\mathrm{i}$ saperi. Si tratta di un tema non solo teoreticamente rilevante, ma anche praticamente urgente:

"L'eccessiva settorialità del sapere - ha affermato Benedetto XVI nella Caritas in veritate -, la chiusura delle scienze umane alla metafisica, le difficoltà del dialogo tra le scienze e la teologia sono di danno non solo allo sviluppo del sapere, ma anche allo sviluppo dei popoli, perché, quando ciò si verifica, viene ostacolata la visione dell'intero bene dell'uomo nelle varie dimensioni che lo caratterizzano. L'allargamento del nostro concetto di ragione e dell'uso di essa è indispensabile per riuscire a pesare adeguatamente tutti i termini della questione dello sviluppo e della soluzione dei problemi socio-economici" (n. 31).

Svolgerò il tema in due momenti: nel primo, richiamerò qualche dato sullo statuto epistemico della teologia, sulla sua singolarità e sull'ambito del conoscere che le è proprio; nel secondo, affronterò la questione del rapporto tra la teologia e gli altri saperi, disegnando alcune coordinate di una "mappa dei saperi” che sia coerente con l'esercizio dell'intelligenza in Cristo. 


\section{Sullo statuto, la singolarità e l'ambito epistemico della teologia}

Una parola sullo statuto e il significato epistemico della teologia cristiana. Illuminanti, nell'indirizzare un approccio pertinente, sono il sostantivo e l'aggettivo che qualificano il sapere della fede. La teologia cristiana, infatti, definisce l'esercizio dell'intelligenza a partire dall'esperienza vissuta della fede, quale corrispondenza libera e ragionevole all'avvento di Dio che si fa escatologicamente presente alla storia in Gesù. Il lemma teologia cristiana, dunque, designa l'esercizio dell'intelligenza a partire e nell'orizzonte del dirsi di Dio all'uomo in Gesù'.

Ciò rappresenta un dato primordiale e specifico dell'esperienza cristiana. Tanto che propriamente non si dà esperienza cristiana senza intelligenza di sé: il dirsi di Dio in Gesù per sé esige la libertà e l'intelligenza in quanto chiamate ad accogliere il dono e a esprimere e comunicare la verità della parola di Dio così rivolta all'uomo. Questa realtà è espressa nel Nuovo Testamento non solo attraverso l'attestazione secondo cui Gesù trasmette le parole che ha ricevuto dal Padre (cf. Gv 3,34; 8,38; 12,48-50; 14,10; 17,6-8), ma - in forma ricapitolativa e definitiva - attraverso l'attestazione secondo cui egli stesso è "la" parola (Lógos) di Dio espressa nella carne della sua umanità e nella vicenda della sua storia (cf. Gv 1,1.14).

Di qui i due compiti di cui la teologia è chiamata a farsi carico:

1) da un lato, esibire l'affidabilità responsoriale dell'evento di Gesù Cristo in conformità al significato che ad esso è riconosciuto nella libertà e nell'intelligenza della fede;

2) dall'altro, esprimere la rilevanza di tale significato nell'intelligenza dell'esistenza umana vissuta nella storia e nel cosmo, secondo le sue molteplici dimensioni.

Nell'un caso come nell'altro è evidente che l'esercizio della teologia si può dare soltanto in correlazione con le diverse forme del sapere dell'uomo:

1) sia perché la giustificazione dell'affidabilità dell'evento di Gesù Cristo nel suo significato di verità presuppone la molteplice pre-comprensione dell'uomo e del mondo offerta dai diversi saperi;

\footnotetext{
${ }^{1}$ Per un'introduzione alla questione mi permetto rinviare al mio Teo-logia. La parola di Dio nelle parole dell'uomo, PUL - Mursia, Roma 1997, $2005^{2}$.
} 
2) sia perché l'articolazione della rilevanza oggettiva di questo significato implica ed esige non solo l'autonomo apporto dei diversi saperi secondo le loro specifiche competenze e i loro specifici oggetti, ma anche, contemporaneamente, la determinazione dell'apporto peculiare che a sua volta la teologia è chiamata a offrire.

Tale assunto ha senz'altro orientato, più o meno esplicitamente e con più o meno successo, la storia della teologia lungo i secoli. A partire dal fatto stesso che essa sia potuta nascere, contestualmente all'attestazione biblica dell'evento di rivelazione di Dio in Gesù, e si sia via via potuta sviluppare.

A esplicitare la portata e le conseguenze di questo assunto, è importante focalizzare la singolarità della teologia cristiana rispetto a due diversi e persino opposti modi d'intendere il luogo e il ruolo epistemici di ciò che, in senso lato, possiamo definire "teologia" nell'ambito dei diversi universi religiosi: e cioè conoscenza di Dio (o del Divino) in riferimento al destino dell'uomo.

Il primo modo è quello di pensare Dio (o il Divino) come talmente altro dall'umano da ritenere alla fine irrilevanti l'esperienza e l'eventuale intelligenza di esso rispetto all'uomo; il secondo è quello di pensare invece Dio (o il Divino) con tale immediatezza e cogenza presente all'uomo e al mondo - senza cioè la corrispettiva mediazione della libertà e dell'intelligenza - da finire col determinarne a priori tutte e singole le espressioni.

Nel primo caso, i diversi saperi dell'uomo non entrano né possono entrare in relazione e tanto meno in conflitto con Dio (o il Divino): che resta per principio avvolto nella "nube della non conoscenza"; nel secondo, i diversi saperi sono determinati integristicamente dal sapere di Dio e pertanto non possono né debbono valere in quanto autonomi. In forma idealtipica, la prima figura è quella dell'agnosticismo, la seconda quella del fondamentalismo, con tutta la sequela delle loro possibili declinazioni.

Lo statuto epistemico della teologia cristiana si distingue radicalmente da entrambi. Per rendersene conto è sufficiente sostare con attenzione sulla singolarità dell'evento di Gesù Cristo accolto nella fede dalla Chiesa. Che cosa significa, infatti, che Dio si dice escatologicamente in Gesù? Almeno tre cose.

a) Innanzi tutto, che l'uomo (e in lui il cosmo) è linguaggio per sé capa$\mathrm{ce}^{2}$ e come tale chiamato a esprimere Dio.

b) In secondo luogo, che la capacità originaria che l'uomo (e il cosmo in lui) è (sono) di esprimere Dio si attua in forma sempre più piena quanto più

${ }^{2}$ Anche questo è significato nell'antica formula che definisce l'uomo capax Dei (capace di Dio). 
l'uomo ri-conosce tale chiamata e vi corrisponde secondo la via e la forma propostegli in Gesù.

c) In terzo luogo, che proprio in questo modo - in quanto cioè l'uomo si riconosce e si fa espressione di Dio in Gesù - Dio è riconosciuto come Dio e l'uomo è riconosciuto/realizzato come uomo nella sua costitutiva, in quanto libera, relazione a Dio: figlio nel Figlio.

Le due verità fondamentali che specificano la fede cristiana non fanno che affermare e articolare questi contenuti. La prima - la verità secondo cui Gesù è vero Dio e vero uomo, "consostanziale" a Dio nella divinità e "consostanziale" agli uomini nell'umanità ${ }^{3}$ - afferma che Gesù è vera e piena espressione dell'uomo in quanto e perché è vera e piena espressione di Dio. La seconda - la verità secondo cui Gesù è il Figlio/Lógos di Dio che è Padre e come tale è la seconda persona della Trinità ${ }^{4}$ - afferma che Gesù è espressione di Dio (divina e umana insieme, distintamente) in quanto è il Figlio/Lógos del Padre fatto carne.

Le due verità centrali della fede cristiana - l'incarnazione e la Trinità disegnano dunque i presupposti e l'orizzonte entro i quali si esplica nella luce dell'evento cristologico il significato del rapporto tra Dio e l'uomo e, di conseguenza, il significato del sapere di/su Dio in rapporto ai saperi di/sull'uomo. Scriveva il pensatore russo Vladimir Solov'ëv:

"La teologia è la scienza divina, ma il Dio dei cristiani si è unito all'umanità con un'unione indissolubile. La teologia del Dio-Uomo non può, dunque, esser separata dalla filosofia e dalla scienza degli uomini"’.

È importante inoltre sottolineare che la verità di fede dell'incarnazione del Figlio/Lógos e quella della Trinità di Dio conoscono un preciso punto

\footnotetext{
${ }^{3}$ Così afferma il Concilio di Calcedonia $(451)$ che ha definito la verità di fede dell'identità divino-umana di Gesù Cristo: "Seguendo i santi padri, all’unanimità noi insegniamo a confessare un solo e medesimo Figlio, il Signore nostro Gesù Cristo, perfetto nella sua divinità e perfetto nella usa umanità, vero Dio e vero uomo, consostanziale al Padre per la divinità, e consostanziale a noi per l'umanità" (DH 301).

${ }^{4}$ E questo il contenuto centrale della fede cristiana in Dio Trinità, espressa in modo sintetico dal Concilio Costantinopolitano II (553): «Chi non confessa che il Padre, il Figlio e lo Spirito Santo hanno una sola natura o sostanza, una sola virtù e potenza, poiché essi sono una Trinità consostanziale, una sola divinità da adorarsi in tre ipostasi o persone, sia anatema. Uno infatti è Dio Padre, dal quale sono tutte le cose; uno il Signore Gesù Cristo, mediante il quale sono tutte le cose; uno è lo Spirito Santo, nel quale sono tutte le cose» (DH 421).

${ }^{5}$ V. Solovèv, La Russia e la Chiesa universale, tr. it., La Casa di Matriona, Milano 1989, 230.
} 
d'incontro che non solo le lega indissolubilmente l'una all'altra, ma al tempo stesso ne illumina reciprocamente l'intelligenza. Si tratta - come sempre ha intuito e professato la Chiesa - dell'evento pasquale di Gesù e, al cuore di esso, del dono di sé sino alla fine, sino all'abbandono da egli vissuto nella pasqua, dono di sé sul quale l'esperienza cristiana ha appuntato con decisione lo sguardo soprattutto nella seconda metà del XX secolo ${ }^{6}$.

L'evento pasquale di Gesù esprime e rivela il significato dell'incarnazione: Dio, in Gesù, si fa uomo all'estremo, sino alla morte, anzi sino a sentirsi "altro" e addirittura "estraneo" rispetto a Dio. In Gesù "appeso al legno" (cf. Gal 3,13) si realizza l'admirabile commercium cantato dalla fede della Chiesa nella contemplazione del Natale: il meraviglioso scambio per cui Dio si fa uomo, affinché l'uomo possa partecipare alla vita di Dio.

Ciò è ontologicamente praticabile perché Dio è in sé l'Amore in cui il Padre si fa l'altro da sé, il Figlio, essendo essi uno e distinti nello Spirito Santo. Dunque, l'incarnazione (spinta sino al suo vertice pasquale) mostra in atto, nella relazione tra Dio e l'uomo, la "legge" di vita in cui s'esprime l'essere di Dio, l'agápe - legge che è principio, forma e destino dell' essere creato nella chiamata a partecipare per dono e in libertà all'essere increato di Dio.

Guardando all'interesse che qui ci occupa, si può affermare che il Crocifisso/ Risorto è il luogo escatologico in cui Dio guarda all'uomo e al cosmo nell'evento del loro diventare, per grazia, ciò che sono chiamati a essere in Gesù; e al tempo stesso la forma che l'umano conoscere è chiamato ad assumere per guardare a Dio com'è in sé e al mondo come è (ed è chiamato a essere) in relazione a Dio.

Ne derivano due conseguenze, a mio avviso decisive per impostare con pertinenza il rapporto tra la teologia e gli altri saperi.

a) La prima discende dal fatto che tra Dio e l'uomo, in Gesù, non vi è né "confusione" né "separazione". Dio e l'uomo - come dice la relazione tra Gesù e il Padre nello Spirito Santo - sono tanto più uno quanto più sono distinti, e viceversa. Il paradigma dell'unità come koinonía dei distinti, che si

\footnotetext{
${ }^{6}$ Cf., in generale, P. Coda, Evento pasquale. Trinità e Storia. Genesi, significato e interpretazione di una prospettiva emergente nella teologia contemporanea, Città Nuova, Roma 1984; "Dio", in Dizionario di Teologia, a cura di G. Barbaglio, G. Bof, S. Dianich, Edizioni San Paolo, Cinisello Balsamo 2002, 407-457; e, specificamente, sull'abbandono in prospettiva biblica, G. Rossé, Il grido di Gesù in croce. Approccio biblico, in "Sophia", 1 (2008/0), 47-60; e, nella prospettiva della sua rilevanza culturale, G.M. Zanghí, Gesù abbandonato maestro di pensiero, Città Nuova, Roma 2008.

${ }^{7}$ Così si esprime il Concilio di Calcedonia, descrivendo il rapporto tra la natura divina e la natura umana nell'unità della persona del Figlio/Lógos (cf. DH 302).
} 
sprigiona dalla fede cristiana, è il paradigma che vede unità e distinzione quali realtà co-originarie, direttamente proporzionali e reciprocamente correlate.

b) La seconda conseguenza discende dal significato integrale che va riconosciuto all'affermazione secondo cui è in Gesù che Dio si dice. Questa affermazione di fede, infatti, significa senz'altro che è singolarmente in lui, in Gesù, che Dio dice se stesso nell'uomo ${ }^{8}$; ma significa al tempo stesso che tutti gli uomini e in definitiva tutto il cosmo sono liberamente chiamati, in Gesù, a farsi in pienezza e verità ciò che sono destinati a essere per creazione: espressione creata di Dio 9 . Il dirsi di Dio in Gesù, in altri termini, è panumano e pancosmico per vocazione, in quanto indirizzato a tutti gli uomini e all'intero cosmo.

Da quanto sin qui detto, si evince il carattere paradossale, a tutta prima, ma a ben vedere straordinariamente suggestivo e illuminante della teologia cristiana. Il fatto è che - essendo fondata nel suo statuto epistemico in Gesù, vero Dio e vero uomo - essa parla di Dio parlando dell'uomo (Gesù) e parla dell'uomo (Gesù) parlando di Dio.

La teologia infatti, per il suo stesso statuto, è chiamata in definitiva a pensare la verità e le condizioni di realizzazione di questa communicatio idiomatum (comunicazione delle proprietà e dei linguaggi) ${ }^{10}$ tra Dio e 1'uomo in Gesù. Essa, in particolare, circoscrive a partire dalla rivelazione i due ambiti del sapere che le sono propri.

a) Il primo è quello del parlare di Dio a partire da Gesù. In questo senso, l'oggetto della teologia è Dio che si rivela in Gesù: un oggetto che - come ha sempre affermato la tradizione della Chiesa ${ }^{11}$ - è in verità sog-

${ }^{8}$ È questo il significato della verità di fede dell'unione ipostatica quale proprietà singolare di Gesù: e cioè il fatto per cui egli è, nell'unità della sua persona, indissolubilmente e distintamente vero Dio e vero uomo.

${ }^{9}$ Secondo quanto afferma l'apostolo Paolo, per cui alla fine Cristo sarà «tutto in tutti» (Col 3,11) e, per Lui e in Lui, anche Dio/Abbà sarà "tutto in tutti" (1Cor 15,28). In questa prospettiva - sottolinea H.U. von Balthasar - «il "confine" posto dal Concilio di Calcedonia a distinzione delle due nature nel Cristo è in fondo non confine che divide, ma possibilità di una diretta unione: di quelle sante nozze fra Dio e la creatura, la cui realizzazione sostanzialmente è rappresentata dal Cristo, dalla Chiesa, dallo Spirito Santo» (Fides Christi, in Sponsa Verbi. Skizzen zur Theologie II, Johannes Verlag, Einsiedeln 1961; tr. it., Morcelliana, Brescia 1985, 41-74, qui 72)

${ }^{10}$ Come noto, lespressione designa, nella teologia dei Padri della Chiesa, il principio per cui - in virtù dell'unità della sua persona - a Gesù vanno distintamente attribuite sia le proprietà della sua divinità sia le proprietà della sua umanità: così che ciò che si dice di lui in quanto Dio si dice anche di lui in quanto uomo, e viceversa.

${ }^{11}$ Cf. San Tommaso d'Aquino, Summa Theologia, I, q. 1, a. 7: "Utrum Deus sit subiectum huius scientiae". 
getto: perché è Dio che dice se stesso in Gesù. La teologia ha il compito di accogliere questa parola da Dio e di esprimerla nelle parole dell'uomo. In questo modo essa, per vocazione, tiene aperto e s'inoltra nello spazio della relazione che dall'uomo rimanda a Dio perché, prima e originariamente, da Dio apre e guarda all'uomo ${ }^{12}$.

Non solo. La teologia, in Gesù, è chiamata a determinare la forma della relazione tra Dio e l'uomo come relazione gratuita e libera di creazione destinata per grazia a compiersi nella figliolanza: l'uomo e il cosmo sono creati per mezzo di Gesù, in vista di lui e perciò in lui sussistono (cf. Col 1,16-17).

Di più ancora. Su questa stessa via, la teologia può e deve parlare di Dio in se stesso, consapevole a un tempo dell'umanità, limitatezza e provvisorietà del proprio parlare ma, in virtù della vera umanità di Gesù, della consistenza e del significato di tale parlare di Dio a partire dall'uomo-Gesù che è il Figlio/Lógos di Dio fatto carne. La summa del suo parlare di Dio in Dio, a partire da Gesù, è la verità della Trinità, e cioè dell'esser uno e trino di Dio in quanto agápe (cf. $1 \mathrm{Gv}$ $4,8.16)^{13}$. Questa verità, concernendo l'essere di Dio, costituisce l'orizzonte di verità - sempre di nuovo da illuminare e approfondire - dell'intera realtà in cui l'uomo (vertice e sintesi del creato visibile) è creato "a immagine $e$ somiglianza" di Dio (cf. Gen 1,26) ${ }^{14}$.

\footnotetext{
${ }^{12}$ Rivolgendosi ai docenti della Facoltà di Teologia dell'Università di Tübingen, Benedetto XVI ha affermato: «Ritengo (...) che la teologia, pur nell'ambito della scientificità, sia richiesta e interpellata sempre anche al di là della scientificità. L'università, l'umanità ha bisogno di domande. Laddove non vengono più poste domande, fino a quelle che toccano l'essenziale e vanno oltre ogni specializzazione, non riceviamo più nemmeno delle risposte. Solo se domandiamo e se con le nostre domande siamo radicali, così radicali come deve essere radicale la teologia, al di là di ogni specializzazione, possiamo sperare di ottenere delle risposte a queste domande fondamentali che ci riguardano tutti. (...) Ma, aggiungerei, per la teologia occorre, oltre il coraggio di domandare, anche l'umiltà di ascoltare le risposte che ci dà la fede cristiana; l'umiltà di percepire in queste risposte la loro ragionevolezza e di renderle in tal modo nuovamente accessibili al nostro tempo e a noi stessi. Così non solo si costituisce l'università, ma anche si aiuta l'umanità a vivere» (21 marzo 2007).

${ }^{13}$ Così si esprime il Catechismo della Chiesa Cattolica: "Il mistero della Santissima Trinità è il mistero centrale della fede e della vita cristiana. È il mistero di Dio in se stesso. È quindi la sorgente di tutti gli altri misteri della fede; è la luce che li illumina. È l'insegnamento più fondamentale ed essenziale nella "gerarchia delle verità" di fede. Tutta la storia della salvezza è la storia del rivelarsi del Dio vero e unico: Padre, Figlio e Spirito Santo, il quale riconcilia e unisce a sé coloro che sono separati dal peccato" (n. 234).

${ }^{14}$ Tutto questo, sia pure in modo iniziale e legato alla cultura del loro tempo, avevano già compreso, in una linea originale di tradizione, Sant'Agostino e, sulla sua scia, San Bonaventura e la teologia francescana, quando vedevano nello spirito dell'uomo e, in genere, in tutte le cose create un'impronta (rispettivamente, imago e vestigium) della Trinità. San Tommaso, soprattutto
} 
b) Il secondo ambito di sapere proprio della teologia è quello del parlare dell'uomo (e in lui del cosmo) a partire da Gesù come parola incarnata di Dio. Non si tratta, dunque, di parlare delle molteplici espressioni dell'uomo (nel suo essere personale e sociale) e del cosmo in se stesse, e cioè nella consistenza propria e specifica del loro essere e delle loro molteplici dimensioni: ciò è compito specifico e autonomo delle diverse forme del sapere umano. Si tratta piuttosto di parlare dell'uomo (e del cosmo) dal punto di vista della sua relazione con Dio in Gesù̀ ${ }^{15}$ : nella triplice prospettiva della protologia (relazione $d a$ Dio), dell'escatologia (relazione $a$ Dio) e dell'ontologia (relazione in Dio). È soprattutto in questo secondo ambito che s'intrecciano i rapporti tra la teologia e le diverse forme del sapere umano, secondo modalità che vanno debitamente determinate $\mathrm{e}$ praticate per evitare gli opposti scogli della "confusione" e della "separazione".

\section{Sul rapporto tra la teologia e gli altri saperi}

Da quanto detto consegue - ecco il secondo momento del nostro percorso - che, in ragione del suo specifico statuto epistemico, la teologia può e deve contribuire a istituire quello spazio vitale e sapienziale (ultimamente fondato

nella sua opera giovanile, il commento alle Sentenze, interpretava la creazione e la storia della salvezza come prolungamento delle processioni trinitarie, rinvenendo nella generazione eterna del Figlio e nell'eterna spirazione dello Spirito la "ratio et causa" della creazione e della redenzione delle creature. La teologia contemporanea va più avanti. E sottolinea che non è sufficiente vedere l'impronta del Dio trinitario nelle singole realtà, nella loro origine e nella loro meta, considerandole però in modo statico e isolate le une dalle altre; quanto piuttosto è necessario interpretarle trinitariamente nel loro dinamico farsi e nel loro vicendevole rapportarsi. In altre parole, alla luce di un Dio che è - come s'esprime la tradizione - reciproco relazionarsi di Persone la cui vita è Amore, bisogna comprendere - come ha scritto K. Hemmerle - che "tutto raggiunge il proprio compimento e realizza la sua propria essenza entrando nella propria relazionalità, nel proprio trascendimento di sé, nel suo possedersi dando se stesso e nell'essere rivolto l'uno all'altro e l'uno per l'altro" (Tesi di ontologia trinitaria. Per un rinnovamento del pensiero cristiano, tr. it., Città Nuova, Roma $\left.1996^{2}, 54\right)$.

${ }^{15}$ Tommaso d'Aquino sottolinea che affermare che Dio è subiectum della teologia significa che "omnia pertractantur in sacra doctrina sub ratione Dei: vel quia sunt ipse Deus; vel quia habent ordinem ad Deum, ut ad principium et finem" (cf. S.Th., I, q. 1 a. 7). Si può dunque dire che la ratio in virtù della quale vengono trattate tutte le realtà in teologia è quella che risiede in Dio stesso: riguarda ciò che pertiene a Dio in quanto Egli è Dio e come tale si rivela e opera in Gesù e nello Spirito Santo. In questo senso, è Dio per primo, ovviamente, il subiectum della teologia, Dio conosciuto in sé e a partire da sé come si rivela in Gesù, in ciò appunto per cui è Dio; ma lo sono anche tutte le realtà create, in quanto la loro ragione ultima è Dio, essendo Egli, in quanto Dio, il principio e il fine, in Gesù, del loro essere e del loro divenire. Le realtà create, in altri termini, vengono trattate nella teologia secondo quella relazione, a loro essenziale, per cui esse non sussistono in sé e per sé, ma da Dio e per Dio in Gesù. 
nell'avvento di Dio nella storia degli uomini che è Gesù) in cui i diversi saperi, ciascuno nell'esercizio del proprio metodo e nell'indagine intorno al proprio oggetto, possano contribuire a promuovere i sentieri molteplici verso la verità tutt'intera dell'uomo e del mondo. In conformità a ciò, la teologia può e deve di concerto intessere un dialogo bilaterale e proficuo per entrambe le parti con i diversi saperi ${ }^{16}$.

Senza pretesa di completezza, e a titolo esemplificativo, mi pare utile spendere qualche parola intorno al rapporto tra la teologia e i seguenti ambiti del sapere: la filosofia, le scienze umane e sociali, le scienze della natura.

a) Innanzi tutto la filosofia. Con la semplificazione necessaria in casi come questo, si può dire che la filosofia descrive l'esercizio dell'intelligenza intorno alla verità di ciò che è (nella nostra esperienza e a partire dalla nostra esperienza): la sua costituzione, il suo senso, il suo destino. Non a caso, pur con differenti interpretazioni, si può con fondatezza affermare che il cuore del sapere filosofico è l'ontologia - il sapere cioè intorno alla verità e al senso dell'essere.

Ora, un tale ambito del sapere umano è necessario e fecondo non solo "prima" (temporalmente e spazialmente) dell'evento della rivelazione di Dio, ma anche e altrettanto "dopo" e in relazione ad esso. E ciò almeno in due direzioni.

La prima: la filosofia è indispensabile per istituire la verità dell'essere dell'uomo e del cosmo come a priori aperto e dinamico in cui si fa percepibile e credibile la parola di Dio. Il che, evidentemente, non significa relativizzare o togliere (aufheben) il valore e il significato della filosofia: poiché l'avvento della parola di Dio, nel suo stesso accadere, non potrà che confermare e, se possibile, offrire una consistenza ancora maggiore a tale a priori.

\footnotetext{
${ }^{16}$ Su questo tema, di recente, cf. il panorama offerto da G. Accordini, Apologia del profilo scientifico. Epistemologia della scienza, epistemologia teologica ed epistemologia della fede, in G. Angelini - S. Macchi (ed.), La teologia del Novecento. Momenti maggiori e questioni aperte, Glossa, Milano 2008, 381- 412, con la rassegna ragionata dei modelli proposti da W. Pannenberg, G. Sauter, B. Lonergan, J. Ladrière. Nello stesso volume si veda anche il contributo di G. Angelini, Teologia, Chiesa e cultura nella stagione postmoderna, (ivi, 695-783), il quale sottolinea lucidamente: «La ricerca interdisciplinare è riconosciuta nominalmente da tutti come necessità imprescindibile; precisarne la consistenza, dire dunque a quali condizioni essa è possibile, appare però compito assai arduo, di fatto eluso. I principi dichiarati dalle singole discipline non paiono predisporre un apparato concettuale idoneo a precisare il senso della ricerca interdisciplinare; mancano infatti di registrare e chiarire il riferimento dei singoli saperi al sapere comune, sotteso alle forme che assume l'intesa reciproca nella vita ordinaria; mentre proprio tale sapere precedente, attestato dalla lingua comune» (ivi, 718).
} 
La seconda: la filosofia risulta indispensabile anche "dopo" e all'interno dell'evento della rivelazione, poiché tale evento propizia e chiede per sé l'intelligenza dell'essere che in sé manifesta e realizza.

Di qui risulta che, sia nella prima sia nella seconda direzione, può e deve darsi reciproco incontro e reciproco scambio tra teologia e filosofia: nel rigoroso rispetto delle loro distinzione e autonomia, ma con l'opportunità praticabile e persino necessaria di un loro reciproco arricchimento ${ }^{17}$.

b) Veniamo alle scienze umane e sociali. Le quali non a caso sono frutto di un'esigenza diventata impellente con la modernità e rispondente, a ben vedere, a un'emergenza nell'articolazione dei saperi umani propiziata, ciascuna a modo proprio, dalla teologia e dalla filosofia. All'origine di tale congiuntura storica penso sia possibile individuare una spinta originaria e progressiva dell'evento cristiano.

Le scienze umane e sociali, infatti, intenzionano la plasticità dinamica dell'essere umano nell'interazione delle distinti dimensioni del suo esprimersi (a livello corporeo, psichico, spirituale) e nella sua costitutiva relazione agli altri - nella città e nel cosmo. Da ciò l'attenzione non solo alla struttura psico-fisica e allo sviluppo dell'essere dell'uomo, ma anche alla mediazione offerta dalle forme simboliche e dalle configurazioni sociali, economiche, politiche in cui si esprime il suo essere nel mondo come comunità locale e universale. Si tratta dunque d'indagare con appropriate metodologie il concreto esprimersi dell'essere umano nella sua corporeità, nella sua libertà, nella sua storicità e nella sua socialità - quali determinazioni intrinseche dell'essere umano acquisite progressivamente alla consapevolezza epistemica in forma specifica nei secoli della modernità.

L'apporto delle scienze umane e sociali si mostra pertanto imprescindibile e costituisce un indispensabile arricchimento per la teologia (così come per la filosofia); mentre a sua volta la teologia, esibendo l'intelligenza della verità dell'uomo nell'orizzonte della sua relazione con Dio in Gesù, è chiamata a offrire i principi e le linee d'interpretazione e discernimento dei dinamismi antropologici e sociali nel loro significato definitivo e come tale incidente assiologicamente sulla loro configurazione e destinazione storica.

\footnotetext{
${ }^{17}$ Cf. su tutto ciò K. Hemmerle, Tesi di ontologia trinitaria, cit., 29-34; e il mio Ontologia trinitaria e sapere delle scienze, in La questione ontologica tra scienza e fede, a cura di P. Coda, Lateran University Press, Roma 2004, 227-239.
} 
c) Da ultimo, le scienze della natura. Il loro ambito d'indagine è il cosmo $\mathrm{e}$, in generale, la struttura e la dinamica, l'origine e lo sviluppo di ciò che esso è e si mostra a livello fisico, chimico, biologico ed ecologico. Anche in questo caso, e ancor prima delle scienze umane e sociali, si è realizzato, a partire dalla fine del Medio Evo, il necessario affrancamento dalla teologia e dalla filosofia, con la messa in opera progressiva di una pluralità di metodi adatti a questo specifico tipo d'indagine e il ritrovamento di specifici oggetti.

Per sé, anche questa volta non si dà conflitto tra il sapere teologico (e filosofico) e quello delle scienze della natura. Come affermava, ancora agli albori del configurarsi teorico e pratico della razionalità scientifica moderna, Cesare Baronio, essa, la scienza moderna, non pretende dire in quale modo si va in cielo, ma come il cielo funziona ${ }^{18}$. D'altra parte - per non fare che un esempio -, la visione cosmologica che è propiziata dalle moderne scienze della natura non può non comportare un correlativo approccio della teologia della creazione, della storia, dell'escatologia.

Il rapporto, difficile eppure ineludibile, tra scienze nel significato moderno del termine e teologia, a proposito della visione del mondo e dell'uomo, si colloca in realtà sullo sfondo dell'intera vicenda della cultura occidentale. La visione del mondo e quella dell'uomo hanno costituito il caso serio dell'incontro/scontro e della reciproca stimolazione tra fede e scienze, da cui sono emerse progressivamente, e sempre più nettamente, l'autonomia e la specificità degli apporti di entrambe per la crescita dell'uomo.

Pur racchiudendo in sé formidabili elementi di novità per l'interpretazione complessiva del reale - basti solo pensare al principio di creazione -, nel percorso della cultura occidentale la rivelazione ebraico-cristiana ha inciso primariamente, e in modo consistente, sulla visione dell'uomo ${ }^{19}$. E ha assunto la visione cosmologica accreditata dalla cultura del tempo combinandola appunto con il principio di creazione, e ritrovando in essa - come hanno notato teologi come Wolfhart Pannenberg ${ }^{20}$ e Hans Urs von Balthasar ${ }^{21}$ - un quadro interpretativo ed espressivo soddisfacente della sua autocomprensione.

\footnotetext{
18 "Spiritui Sancto mentem fuisse nos docere quomodo ad coelum eatur, non quomodo coelum gradiatur" (frase di C. Baronio citata da Galileo nella lettera a Cristina di Lorena).

${ }^{19}$ Come nota A. Masani nella sua opera La cosmologia nella storia fra scienza, religione e filosofia, La Scuola, Brescia 1996, 39, dove parla di un "capovolgimento di interessi culturali".

${ }^{20}$ Cf. W. Pannenberg, Epistemologia e teologia, tr. it., Queriniana, Brescia 1975, 295.

${ }^{21}$ Cf. H.U. von Balthasar, Solo l'amore è credibile, tr. it., Borla, Roma 1977, 17-32.
} 
E tanto la fede s'è identificata con la visione cosmologica promossa da tale inedita sintesi, da avvertire come destabilizzanti le innovazioni nella visione cosmologica introdotte successivamente, a partire dalla modernità. Le quali, paradossalmente, hanno però costretto la teologia non solo a rendersi conto della specificità della visione di fede rispetto ai saperi delle scienze, ma, in questa distinzione, a recuperare la coscienza delle proprie virtualità in ordine a una visione del mondo in cui convergano le diverse prospettive e $\mathrm{i}$ diversi apporti delle scienze e della fede ${ }^{22}$.

La rapida rassegna, appena abbozzata in forma orientativa, circa il rapporto tra il sapere di Dio e alcune fondamentali forme del sapere dell'uomo, porta a due considerazioni: l'una di carattere storico, l'altra di carattere teoretico.

a) Dal punto di vista storico, mi pare risulti chiaro che da una parte l'evento di Gesù Cristo, dall'altra gli sviluppi conosciuti nell'ambito del sapere umano nella cultura dell'Occidente, hanno finito col disegnare una nuova mappa dei saperi. Sia perché è venuta a costituirsi, in senso proprio, la teologia cristiana come sapere della relazione tra Dio e l'uomo che è avvenuta e avviene in Gesù mediante l'azione incessante e inesauribile del suo Spirito ${ }^{23}$; sia perché l'affermarsi della consistenza propria e della storicità dell'uomo e della struttura specifica e dello sviluppo del cosmo hanno via via esigito l'aprirsi di autonomi spazi d'indagine mediante metodi appropriati.

Tutto ciò (non si tratta infatti di poca cosa) non ha potuto non suscitare accesi dibattiti e persino aspre guerre di competenza sfociate persino nella reciproca scomunica dall'orizzonte del sapere autentico. Si pensi, in prima battuta, alla dialettica tra la tradizione della filosofia greca e l'intelligenza della Parola della rivelazione che ha caratterizzato i primi secoli dell'era cristiana; $\mathrm{o}$, in tempi a noi più vicini, ai conflitti che si sono accesi tra la teologia e le scienze moderne intorno alla questione galileiana e a quella dell'evoluzione.

b) Dal punto di vista teoretico, tutto ciò comporta un passo nuovo che sono oggi chiamati a compiere sia il sapere di Dio sia i saperi dell'uomo. Pena, da un lato, l'estraniazione della teologia dai processi conoscitivi e dalle sfide antropologiche che investono l'umanità con pervasività e urgenza crescenti e,

\footnotetext{
${ }^{22}$ Cf. P. Coda, Teologia trinitaria della creazione e interpretazione scientifica del reale, in "Lateranum”, LXVIII (2002/1), 23-41.

${ }^{23}$ Cf. Gv 16,13: "Quando verrà lo Spirito di verità, egli vi guiderà alla verità tutta intera, perché non parlerà da sé, ma dirà tutto ciò che avrà udito e vi annunzierà le cose future”.
} 
dall'altro, lo svuotamento di senso e di verità per l'uomo degli altri saperi. Un segnale forte in questa direzione, da parte della coscienza cristiana, è venuto da teologi come A. Rosmini nel XIX secolo, K. Rahner, B. Lonergan e W. Pannenberg nel XX, e in forma ecclesialmente rilevante dal Concilio Vaticano II. Benedetto XVI, dal canto suo, ha più volte colto e indicato in questo compito una delle priorità della cultura d'ispirazione cristiana in fedeltà alla consegna del vangelo di Gesù e a servizio della crescita dell'umanità ${ }^{24}$.

Si tratta, nel rispetto dell'identità e del metodo di ogni sapere, di muovere dal condiviso presupposto secondo cui lo statuto epistemico di ogni scienza non riguarda soltanto le condizioni interne per un suo corretto attuarsi, ma anche quelle relazionali relative al rapporto di essa con le altre forme del sapere, che esprimono ciascuna - al proprio specifico livello - qualcosa della persona e attingono ciascuna, per la sua parte, qualcosa di vero circa la realtà ${ }^{25}$. Di qui si fa possibile approdare a una conoscenza convergente e dinamicamente articolata della realtà, in uno spazio epistemico relazionale dove ogni sapere, aprendosi con il proprio metodo e i propri contenuti agli altri saperi, possa adeguatamente esprimersi e offrire il proprio contributo al progetto comune. Alimentando così un autentico dialogo e una libera cooperazione tra i saperi, che tali appunto sono in quanto operanti nell'orizzonte di una razionalità "ampia" perché fondata e illuminata, in radice, da quel Lógos fatto carne, crocifisso e risorto da cui scaturisce e verso cui tende, nel soffio dello Spirito, ogni autentica ricerca della verità.

Di qui la necessità di dar vita a comunità di formazione, di studio e di ricerca in cui anche culturalmente prenda forma quel «soggetto più grande» - per dirla con le parole di papa Ratzinger ${ }^{26}$ - che si costituisce in virtù dell'esperienza

${ }^{24}$ Occorre - egli ha affermato - "allargare gli spazi della nostra razionalità, riaprirla alle grandi
questioni del vero e del bene, coniugare tra loro la teologia, la filosofia e le scienze, nel pieno
rispetto dei loro metodi propri e della loro reciproca autonomia, ma anche nella consapevolezza
dell'intrinseca unità che le tiene insieme. È questo un compito che sta davanti a noi, un'avventura
affascinante nella quale merita spendersi, per dare nuovo slancio alla cultura del nostro tem-
po e per restituire in essa alla fede cristiana piena cittadinanza" (Discorso ai partecipanti al IV
Convegno Nazionale della Chiesa Italiana, Verona, 19 ottobre 2006). Cf. anche Benedetto XVI,
Fede, ragione e università. Ricordi e riflessioni. Discorso ai rappresentanti della scienza presso
l'Università di Regensburg, 12 settembre 2006; e sul suo significato il mio articolo Il significato e
il percorso del Forum alla luce della "lectio magistralis" di Benedetto XVI a Regensburg, in "PATH",
7 (2008/1): Per un nuovo incontro tra fede e logos, 9-23.
${ }^{25}$ Cf. il mio Teo-logia, cit., 59-60.
${ }^{26}$ Cf. Benedetto XVI, Discorso ai partecipanti al IV Convegno Nazionale della Chiesa Italiana, 
di fede, ma è aperto e accessibile a chiunque si ponga in ascolto della verità: comunità nelle quali la relazione agapica, nello Spirito di Gesù, tra le persone comunichi il proprio timbro alla relazione fra le discipline.

\section{Per attingere insieme il "pensiero di Cristo"}

Utopia? Penso piuttosto che il nostro tempo esiga il coraggio delle grandi visioni che si alimentano alla sorgente sempre viva del Vangelo e che, proprio per questo, sanno intercettare nella loro profondità le istanze che sorgono incalzanti dai "segni dei tempi".

Che cosa mai avrà voluto dire, ad esempio, l'apostolo Paolo in quel passo provocante della prima lettera ai Corinzi (cf. 1Cor 2,9-16) dove testimonia ch'è ormai possibile - e definitivamente reale - albergare nel proprio pensare il "noûs" di Cristo stesso? E cioè l'intelligenza in cui le profondità del mistero di Dio non stanno più relegate, nell'indifferenza o nella nostalgia, in un'inaccessibile distanza, ma si offrono a un conoscerle e a un dispiegarle nella loro incidenza storica che nel dirle non le cattura, snaturandole, ma ne gode qualcosa di vero e sostanzioso nella convivialità di una ricerca e di uno scambio sempre nuovi e sempre diversi.

Il discorso di Paolo è stringente. Come solo lo spirito dell'uomo - egli argomenta - può conoscere che cosa veramente passi nel suo cuore e nella sua mente, così solo lo Spirito di Dio "può scrutare le profondità di Dio". Dio dimora infatti segregato irrimediabilmente nella "nube della non conoscenza" per chi è altro da lui. A meno che Egli - e in ciò, a ben vedere, sta l'inaudito del lógos di Gesù, anzi del Lógos fatto carne, crocifisso e risorto che è Gesù - gli conceda e gli consegni il suo stesso Spirito. Come avviene nella carne crocifissa di Gesù, il quale nel suo

Verona, 19 ottobre 2006: “Non sono più io che vivo, ma Cristo vive in me" (Gal 2,20). È stata cambiata così la mia identità essenziale e io continuo ad esistere soltanto in questo cambiamento. Il mio proprio io mi viene tolto e viene inserito in un nuovo soggetto più grande, nel quale il mio io cè di nuovo, ma trasformato, purificato, "aperto" mediante l'inserimento nell'altro, nel quale acquista il suo nuovo spazio di esistenza. Diventiamo così "uno in Cristo" (Gal 3,28), un unico soggetto nuovo, e il nostro io viene liberato dal suo isolamento. "Io, ma non più io": è questa la formula dell'esistenza cristiana fondata nel Battesimo, la formula della risurrezione dentro al tempo, la formula della "novità" cristiana chiamata a trasformare il mondo. Qui sta la nostra gioia pasquale». La determinazione delle qualità esistenziali ed epistemiche del "soggetto" adeguato di tale conoscenza richiederebbe uno sviluppo specifico; rinvio per intanto al mio Sul soggetto della teologia alla luce del carisma dell'unità, in "Nuova Umanità", XXII, n. $132(2000 / 6), 869-893$ 
morire vissuto come agápe "consegna lo Spirito" (cf. Gv 19,30). È precisamente per questa consegna - conclude Paolo - che «noi abbiamo il noûs di Cristo".

Dunque, la "nube della non conoscenza" è squarciata, definitivamente: com'è avvenuto per il velo del tempio di Gerusalemme nell'atto stesso del morire sulla croce del Lógos fatto carne. Dal recesso del sancta sanctorum la presenza di Dio corre verso le tende degli uomini: perché il Lógos incarnato e crocifisso ha posto la sua tenda in mezzo ad essi. In mezzo agli ultimi, agli emarginati e agli scartati. Sul calvario che ormai ha l'estensione del mondo, perché con tutti e con ciascuno Gesù si è "fatto uno" per aprici in sé gli uni verso gli altri nello spazio di quell'amore che è segno e frutto della risurrezione.

Tutti, nessuno escluso, siamo per Gesù in Dio, e Dio è in noi. Ma questo dono - cháris - non si realizza, e cioè non si fa realtà storicamente vissuta $\mathrm{e}$ testimoniata anche nel pensare, se non quando la forma del Lógos crocifisso diventa la forma del nostro pensare Dio e l'uomo là dove essi ora sono: in quel "luogo" cui accediamo, ogni volta in modo nuovo, andando l'uno verso l'altro e così realmente accogliendoci. E cioè arrischiandoci, sino in fondo, in una parola e in un ascolto fatto di reciprocità senza residui e senza condizioni. È lì che attingiamo e ospitiamo, insieme, il Lógos che nello Spirito si fa nostra carne. La verità di Dio che è Trinità - Padre, Figlio/Lógos e Spirito Santo - accade nella verità, che così si va facendo, del nostro essere e del nostro pensare in dialogo gli uni con gli altri ${ }^{27}$.

Il "perché?" nudo, disarmato e arrischiato del Cristo abbandonato sulla croce, è il luogo d'incontro che non appartiene solo al sapere di Dio o solo ai saperi dell'uomo. Siamo noi, piuttosto, che apparteniamo ad esso e che, appartenendogli nel rischio perseverante e sempre nuovo della verità e della libertà, possiamo tornare in forma nuova ad appartenerci gli uni gli altri nella tenerezza disarmata e forte di quell'amore che è la presenza di Dio in noi, primizia e caparra di "cieli nuovi e terra nuova".

\section{Referências Bibliográficas}

ACCORDINI, G., "Apologia del profilo scientifico. Epistemologia della scienza, epistemologia teologica ed epistemologia della fede". In: G. Angelini - S. Macchi (ed.), La teologia del Novecento. Momenti maggiori e questioni aperte, Glossa, Milano 2008, 381- 412.

${ }^{27}$ Cf. G.M. Zanghí, Parole theofore, in "Nuova Umanità, XIX, n. 110 (1997/2), 189-202. 
ANGELINI, G., "Teologia, Chiesa e cultura nella stagione postmoderna". In: G. Angelini - S. Macchi (ed.), La teologia del Novecento. Momenti maggiori e questioni aperte, Glossa, Milano 2008, 695-783.

BALTHASAR, H. U., "Fides Christi". In: Sponsa Verbi. Skizzen zur Theologie II. Einsiedeln: Johannes Verlag, 1961 [tr. it., Morcelliana, Brescia 1985, 41-74].

BALTHASAR, H. U., Solo l'amore è credibile [tr. it.]. Roma: Borla, 1977.

BENEDETTO XVI, “Fede, ragione e università. Ricordi e riflessioni”. Discorso ai rappresentanti della scienza presso l'Università di Regensburg, 12 settembre 2006.

BENEDETTO XVI., Discorso ai docenti della Facoltà di Teologia dell’Università di Tübingen, 21 marzo 2007.

BENEDETTO XVI., Discorso ai partecipanti al IV Convegno Nazionale della Chiesa Italiana, Verona, 19 ottobre 2006.

CODA, P., "Dio". In: Dizionario di Teologia, a cura di G. Barbaglio, G. Bof, S. Dianich. Cinisello Balsamo: San Paolo, 2002, 407-457.

CODA, P., "Il significato e il percorso del Forum alla luce della lectio magistralis di Benedetto XVI a Regensburg". In: PATH 7 (2008/1), 9-23.

CODA, P., "Ontologia trinitaria e sapere delle scienze". In: La questione ontologica tra scienza e fede (a cura di P. Coda). Roma: Lateran University Press, 2004, 227-239.

CODA, P., "Sul soggetto della teologia alla luce del carisma dell'unità". In: Nuova Umanità XXII, n. 132 (2000/6), 869-893.

CODA, P., "Teologia trinitaria della creazione e interpretazione scientifica del reale". In: Lateranum LXVIII (2002/1), 23-41.

CODA,P., Eventopasquale. TrinitàeStoria. Genesi, significatoeinterpretazione di una prospettiva emergente nella teologia contemporanea. Roma: Città Nuova, 1984.

CODA, P., Teo-logia. La parola di Dio nelle parole dell'uomo. Roma: PUL Mursia, 1997, 2005².

HEMMERLE, K., Tesi di ontologia trinitaria. Per un rinnovamento del pensiero cristiano [tr. it.]. Roma: Città Nuova, $1996^{2}$.

MASANI, A., La cosmologia nella storia fra scienza, religione e filosofia. Brescia: La Scuola, 1996.

PANNENBERG, W., Epistemologia e teologia [tr. it.]. Brescia: Queriniana, 1975.

ROSSÉ, G., "Il grido di Gesù in croce. Approccio biblico". In: Sophia 1 (2008/0), 47-60. 
SOLOV’ËV, V., La Russia e la Chiesa universale, [tr. It]. Milano: La Casa di Matriona, 1989.

TOMMASO d'AQUINO Summa Theologia, I, q. 1 a. 7.

TOMMASO d'AQUINO, Summa Theologia, I, q. 1, a. 7.

ZANGHÍ, G. M., "Parole theofore". In: Nuova Umanità XIX, n. 110 (1997/2), 189-202.

ZANGHI, G. M., Gesù abbandonato maestro di pensiero. Roma: Città Nuova, 2008.

\section{Piero Coda}

(Cafasse, Torino 1955) è Preside dell'Istituto Universitario Sophia

"Per una cultura dell'unità" promosso dal Movimento dei Focolari (Loppiano, Incisa in Val d'Arno - Firenze) dove insegna teologia sistematica. Impegnato a livello internazionale nel dialogo tra fede e cultura, cristianesimo e religioni, è Presidente dell'Associazione Teologica Italiana. Da segnalare tra i suoi libri: Evento pasquale. Trinità e Storia (Città Nuova, 1984); Il negativo e la Trinità: ipotesi su Hegel (Città

Nuova, 1987); Il Logos e il Nulla. Trinità, religioni, mistica (Città Nuova, 2003, 20042); Sul luogo della Trinità: rileggendo il "De Trinitate" di Agostino (Città Nuova, 2008); Dalla Trinità. L'avvento di Dio tra storia e profezia (Città Nuova, 2011); e con G. Filoramo, Dizionario del cristianesimo, 2 voll. (Utet, 2006).

Artigo Recebido em 10/08/2011

Artigo Aprovado em 14/10/2011 